\title{
STAT3 and EGFR target resistance
}

The overexpression of EGFR is an established target in a number of cancers, and agents targeting this protein have been approved for the treatment of advanced-stage head and neck squamouscell carcinoma (HNSCC), colorectal cancer and non-small-cell lung cancer. Unfortunately, intrinsic or acquired resistance to these agents means that the efficacy of these agents is limited. Now, a team lead by Jennifer Grandis has revealed that targeting the protein STAT3 can enhance the tumour response to targeted EGFR therapy.

Grandis describes the rationale behind this work: "we previously found that STAT3 activation can mitigate sensitivity to an EGFR tyrosine kinase inhibitor in vitro. We therefore hypothesized that STAT3 activation may contribute to EGFR inhibitor resistance and chose to test that hypothesis in HNSCC." To assess this theory, the team used HNSCC cells lines and a mouse xenograft model of acquired cetuximab resistance. Grandis explains, "to target STAT3 we elected to use a decoy oligonucleotide that we recently demonstrated had pharmacodynamic activity in a phase 0 trial in HNSCC patients." In both of the models, treatment with the STAT3 oligonucleotide reduced cellular viability. In the mouse model, dual targeting of EGFR and STAT3 significantly enhanced the antitumour effects of the agents.

The importance of these results were explained by Grandis: "biopsies from HNSCC patients whose tumours recurred following cetuximab therapy demonstrated increased expression of STAT3, underscoring the potential clinical significance of the findings."

\section{Rebecca Kirk}

Original article Grandis, J. R. et al. Targeting STAT3 abrogates EGFR inhibitor resistance in cancer. Clin. Cancer Res. doi:10.1158/1078-0432.CCR-12-0792 